\title{
Role of quality control circle in sustained improvement of hand hygiene compliance: an observational study in a stomatology hospital in Shandong, China
}

Peng Chen ${ }^{1,2,3+}$, Ting Yuan ${ }^{4 \dagger}$, Qinfeng Sun ${ }^{2,3}$, Lili Jiang ${ }^{2,3}$, Hongmin Jiang ${ }^{2,3}$, Zhenkun Zhu ${ }^{2,3}$, Zexin Tao ${ }^{5,6}$, Haiyan Wang ${ }^{5,6}$ and Aiqiang $\mathrm{Xu}^{1,5,6^{*}}$

\begin{abstract}
Background: Hand hygiene is an important element of the WHO multimodal strategy for healthcare-associated infection control, whereas compliance of hand hygiene among healthcare workers (HCWs) remains a challenge to sustain. In order to increase the hand hygiene compliance of HCWs, a quality control circle (QCC) program was carried out in our hospital, and the plan-do-check-act (PDCA) method was applied for 12 months.

Findings: Hand hygiene compliance rates improved over time, with significant improvement between preintervention (60.1\%) and postintervention (97.2\%) periods $(P<0.001)$. Nurses $(88.3 \%)$ exhibited higher compliance than dentists (87.3\%), and female (88.4\%) HCWs were more likely to perform hand hygiene than males (85.6\%), both $P<0.001$. Overall hand hygiene compliance and observance of the five indications exhibited significant linear increases over time $(P<0.005)$.

Conclusion: This study highlights the success of a multifaceted intervention, conducted by QCC program and PDCA method, which led to a significant improvement of hand hygiene compliance. Though training is the most basic intervention element, surveillance, evaluation and feedback should be explored as additional interventions to ensure that hand hygiene compliance is achieved and sustained at high levels.
\end{abstract}

Keywords: Hand hygiene, Quality control circle, Healthcare-associated infection, Healthcare workers

\section{Background}

Quality control circle (QCC), first established in Japan in 1962, has been widely used in medical and healthcare fields in Germany, Austria and Thailand [1-3]. In 2001, QCC was introduced to medical institutions in China, aiming to increase the quality of medical service by improving medical workers' awareness of spotting and solving medical problems [4-6]. Healthcare-associated infection (HAI) have been identified as a major preventable complication for inpatient care, and hand hygiene

\footnotetext{
* Correspondence: aqxuepi@163.com

${ }^{\dagger}$ Equal contributors

${ }^{1}$ Department of Epidemiology, School of Public Health, Shandong University, Jinan, Shandong, People's Republic of China

${ }^{5}$ Shandong Provincial Key Laboratory of Infectious Disease Control and

Prevention, Jinan, Shandong, People's Republic of China

Full list of author information is available at the end of the article
}

was recommended as one of the most effective strategies to prevent HAI [7]. Hand hygiene is defined as any action of hand cleansing, including practices such as antiseptic hand washing, antiseptic handrubbing, hand washing, hygienic handwash, surgical handscrubbing, etc. [8]. However, the compliance of hand hygiene is generally poor despite a variety of measures were proposed to improve it [9-11]. The World Health Organization (WHO) launched the global hand hygiene campaign in 2004 to improve hand hygiene compliance, which included five indications of before patient contact, before an aseptic task, after body fluid exposure risk, after patient contact and after contact with patient surroundings. The evaluation and feedback of hand hygiene performance are important elements of this campaign. 
In 2013, a QCC program was carried out in department of implant dentistry in Stomatology Hospital of Shandong University, aiming to improve the hand hygiene compliance of healthcare workers (HCWs) by active monitoring using covert observation. Patients treated in this department were all outpatients with dental implantation treatment, and no intravenous line or other indwelling device was applied. The program was reviewed, revised and promoted monthly. This was the first time that the QCC program was explored for the improvement of hand hygiene compliance in our hospital, and it also promoted the application and practice of advanced tools for the quality management and control.

\section{Methods}

The QCC program was launched based on the QCC theory [12], and the basic steps of QCC program were outlined in Additional file 1. The roles of QCC program included project director, counselor, circle head and circle members, which were acted by infection control officer, charge nurse, department head and the rest dentists and nurses, respectively. The program started with the circle head and members understanding the status quo of hand hygiene compliance and investigating the reasons through brainstorming. A fishbone diagram demonstrated the possible reasons for low hand hygiene compliance in this program (Fig. 1). The QCC program was revised and promoted monthly in accordance with plan-do-check-act (PDCA) cycle. Hand hygiene compliance was summarized and evaluated by the project director, and periodic feedback was implemented to all QCC members at the circle meeting each month. An agenda of the circle meeting was showed in Additional file 2.

Intervention measures were then analyzed and modified through quality management approaches. Firstly, hand hygiene products included an alcohol based hand rub agent and liquid soap were increased to at least one for each dental unit to improve the accessibility. Secondly, establishing a covert observer unit was a necessary component to investigate the hand hygiene compliance by unbiased covert observation. Moreover, circle meeting was the most important form to promote the smooth execution of QCC program and was held at least once a month, with an activity log was maintained. At the QCC meeting, hand hygiene compliance was posted up and red flags were awarded to the exceptional individuals. Also, punishments such as paying a fine would be executed to the noncompliant HCWs to hold the accountable for their practices.

We invited medical students to serve as the covert observers during their clinical clerkships. A standard data form was used to record the six hand hygiene compliance, which included WHO recommended indications [8]. As the failure to change gloves between clinical procedures would increase the risk of cross-transmission [13], the indication of after removing gloves was added in our study. All the data was analyzed with SPSS version 18.0, assuming a type I error of $\alpha=0.05$. The Chi square test was used to test the statistical difference in hand hygiene compliance by professional category and gender. Cochran Armitage trend test was used to analyze the linear trend of hand hygiene compliance over time, and the processes were illustrated in Additional file 3.

\section{Results}

From September 2013 to August 2014, a total of 6681 hand hygiene opportunities were detected by direct observation in department of implant dentistry, and 5869 hand hygiene actions were recorded. Kolmogorov-

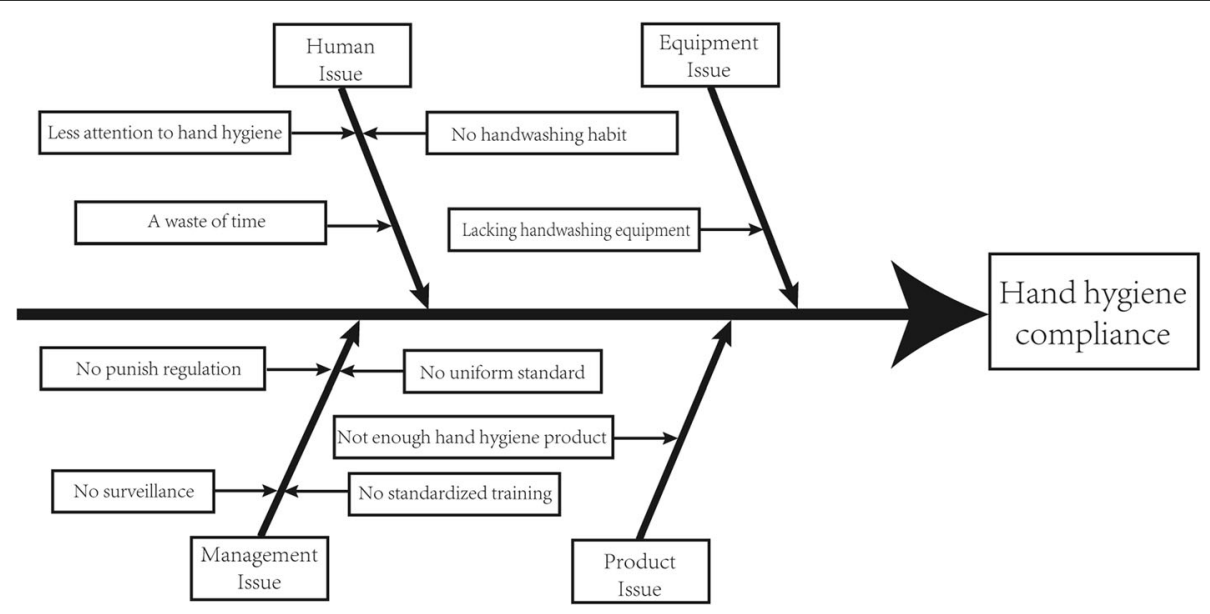

Fig. 1 The reasons for low hand hygiene compliance demonstrated in a fishbone diagram 
Smirnov method showed that the data in each month were subject to a normal distribution, and the mean was used to represent the average compliance during the QCC program. The results of Chi square test revealed that nurses (88.3\%) exhibited higher hand hygiene compliance than dentists (87.3\%), and the female (88.4\%) were more likely to perform hand hygiene than the male (85.6\%), both $P<0.001$ (Table 1). Monthly hand hygiene compliance and the respective $95 \%$ confidence interval were displayed in Fig. 2. During the QCC program, 436 to 705 hand hygiene opportunities were calculated monthly by the covert observers, with an average of 556.7 per month. The hand hygiene compliance was $60.1 \%$ as the QCC program was launched, and eventually the compliance was stabilized with an average rate of $97.5 \%$ for the last three months. Cochran Armitage trend test was applied to evaluate the linear trend of hand hygiene compliance, and the result showed that the compliance exhibited a significant linear increase over time $\left(\chi^{2}=515.833, P<0.005\right)$.

Among the six hand hygiene indications, after body fluid exposure risk, before an aseptic task, after contact with patient surroundings and after removing gloves showed higher compliances ranging $87.7-100 \%, 87.4-100 \%, 84.6-100 \%$ and $82.9-97.2 \%$ (Fig. 3), respectively. However, the compliance of indications such as before patient contact and after patient contact were extremely low, only 22.4 and $37.0 \%$ of hand hygiene opportunities were detected to perform hand hygiene at the beginning of the QCC program. The most important cause of the difference may attribute to the less attention to hand hygiene in the clinical practice. For this reason, HCWs did not develop the handwashing habit in the initial of QCC program, and showed low compliance with hand hygiene before and after patient contact. On the contrary, the hand hygiene compliance was high as some clinical practices were performed, such as after body fluid exposure risk, before an aseptic task. Hence, interventions such as paying a fine were executed to the noncompliant HCWs, and the compliance of before (and after) patient contact raised to $80.2 \%$ (87.9\%) in January 2014 (December 2013). The results of Cochran Armitage trend test were shown in Table 1. All these hand hygiene indications exhibited significant linear increases except the indication of after contact with patient surroundings, which the $P$ value $>0.05$.

\section{Discussion}

Hand hygiene has been identified as the most significant, modifiable cause of HAI, yet hand hygiene compliance is negative in China [14-16]. In our hospital, variety of hand hygiene interventions have been explored in the hospital setting, hand hygiene behavior was surveilled every month and correction reports were released. However, hand hygiene adherence behavior is less than expected among HCWs and the compliance is still a challenge. In 2013, the QCC was explored to improve hand hygiene compliance, and HCWs were encouraged to participate in the management of hand hygiene program. In our study, many of the ideas suggested by QCC members were taken into consideration and multifaceted interventions were applied to improve hand hygiene compliance. For the promotion of hand hygiene, the acceptance and implementation are the most critical. In this QCC program, the full participation of HCWs was essential to implement hand hygiene behavior in their clinical practices. Also, the surveillance-evaluation-

Table 1 Descriptive analysis of hand hygiene opportunities and actions among different parameters

\begin{tabular}{|c|c|c|c|c|c|}
\hline Parameter & Opportunity & Action & Compliance & $x^{2}$ & $P$ \\
\hline Total & 6681 & 5869 & $87.8 \%$ & & \\
\hline \multicolumn{6}{|l|}{ Professional category } \\
\hline Dentists & 3046 & 2659 & $87.3 \%$ & 51.927 & $<0.001$ \\
\hline Nurses & 3635 & 3210 & $88.3 \%$ & & \\
\hline \multicolumn{6}{|l|}{ Gender } \\
\hline Male & 1309 & 1121 & $85.6 \%$ & 2470.883 & $<0.001$ \\
\hline Female & 5372 & 4748 & $88.4 \%$ & & \\
\hline \multicolumn{6}{|l|}{ Hand hygiene indications $^{\mathrm{a}}$} \\
\hline 1. Before patient contact & 1410 & 1079 & $76.5 \%$ & 468.466 & $<0.005$ \\
\hline 2. Before an aseptic task & 991 & 946 & $95.5 \%$ & 24.058 & $<0.005$ \\
\hline 3. After body fluid exposure risk & 873 & 833 & $95.4 \%$ & 26.836 & $<0.005$ \\
\hline 4. After patient contact & 1218 & 1019 & $83.7 \%$ & 169.959 & $<0.005$ \\
\hline 5. After contact with patient surroundings & 752 & 720 & $95.7 \%$ & 3.361 & $>0.05$ \\
\hline 6. After removing gloves & 1437 & 1272 & $88.5 \%$ & 67.367 & $<0.005$ \\
\hline
\end{tabular}

${ }^{\mathrm{a}}$ All indications were recommended by the WHO except after removing gloves 


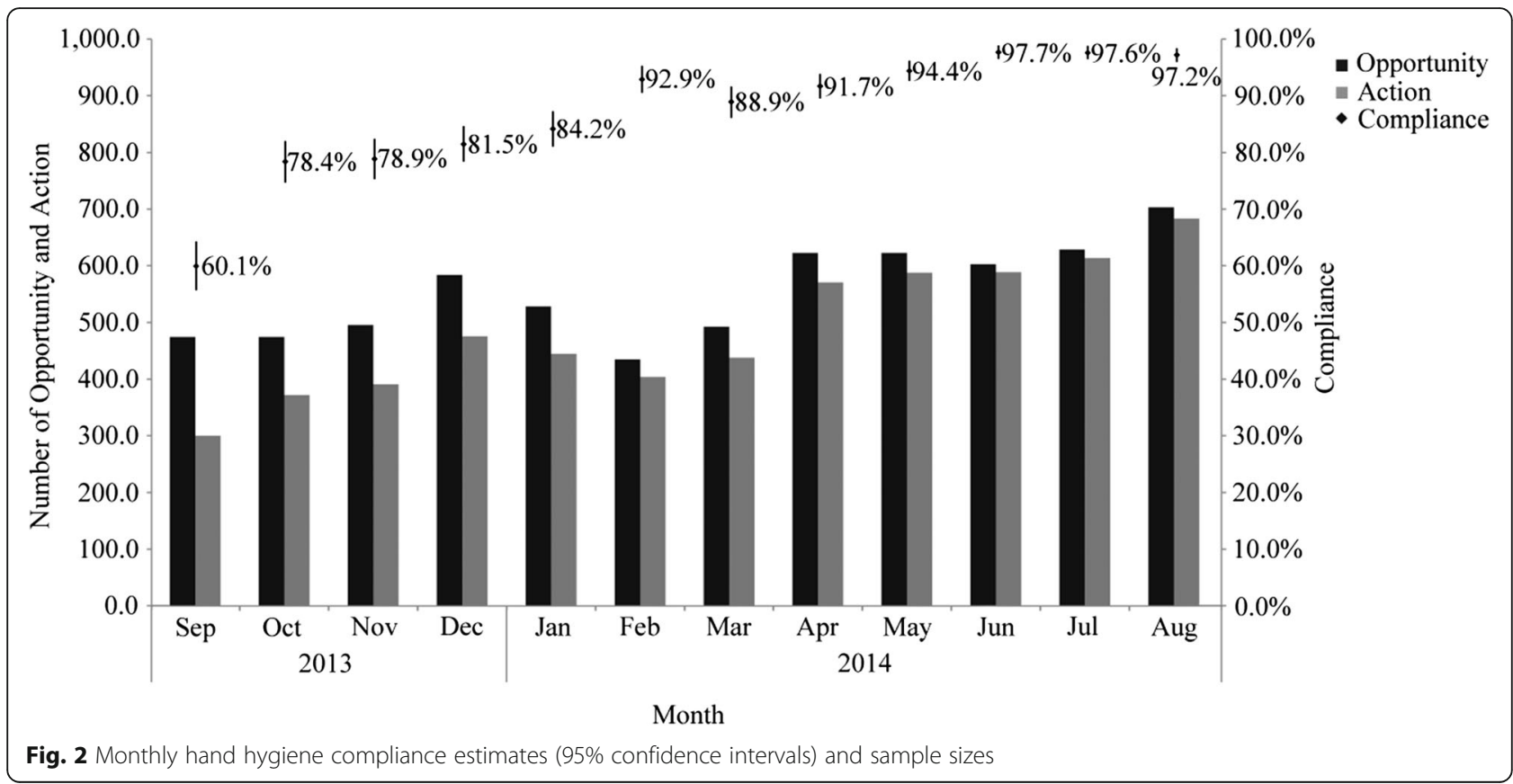

feedback-intervention model should be recommended for a high, sustained level of hand hygiene compliance.

According to the management mode of QCC, continuous monitoring is necessary for the sustained improvement of hand hygiene compliance. Hence, the covert observers were invited to record the hand hygiene compliance aperiodically and the data was summarized and evaluated at the circle meetings. Although the hand hygiene compliance expressed a significant increase in the covert observation, the dentists and male HCWs should be noted as they showed low compliance than the nurses and the female respectively. Meanwhile, the fact that the compliances of before patient contact and after patient contact were extremely low also revealed these indications required more monitoring and intervention as necessary. Of note, various hand hygiene compliances in Fig. 2 showed a drop between December 2013 and January 2014. The main reason for our analysis should be the coming Chinese New Year holiday in February 2014. In such case, interference from other work decreased hand hygiene compliance, and HCWs paid less attention to their hand hygiene behaviors in medical practices. Hence, an additional intervention which assuming corresponding responsibility to the circle head was implemented. Consequently, the hand hygiene program is a long, evolutionary process, and improvement of hand hygiene compliance will be difficult to achieve without systemic and multifaceted interventions.

This study was conducted at a major teaching stomatology hospital, and it had several strengths and limitations. The strengths were as follows. First, the QCC theory was used for the improvement of hand hygiene program, and multifaceted strategies were used. Second, covert observers were chosen to record hand hygiene opportunities and actions, and the HCWs were not aware of the covert observers. The advantage of using covert observation can avoid the Hawthorne effect $[17,18]$ and maintain patient privacy. Third, this study included a very large number of hand hygiene opportunities and actions. Additionally, we analyzed separately by professional category and gender, and significant differences were observed in these parameters. These help to pinpoint areas of strength and weakness in hand hygiene program. However, there were several limitations. One limitation of the method used to measure hand hygiene compliance could lead to selection bias or information bias if the covert observers selected HCWs non-randomly or recorded incorrectly [19, 20]. In anticipation of this bias, we designed a large sample size, and all covert observers were trained and participated in an examination before monitoring hand hygiene behavior. Also, the timing and appropriate technique components of compliance should also be measured and a scale up of this QCC program in other departments is still necessary.

\section{Conclusions}

The improvement of hand hygiene compliance is a worldwide program, and the promotion of hand hygiene behavior needs a long-term effort. QCC can inspire the $\mathrm{HCWs}$ to participate in the management of hand hygiene program, systemic and multifaceted interventions will be more effective in improving hand hygiene 

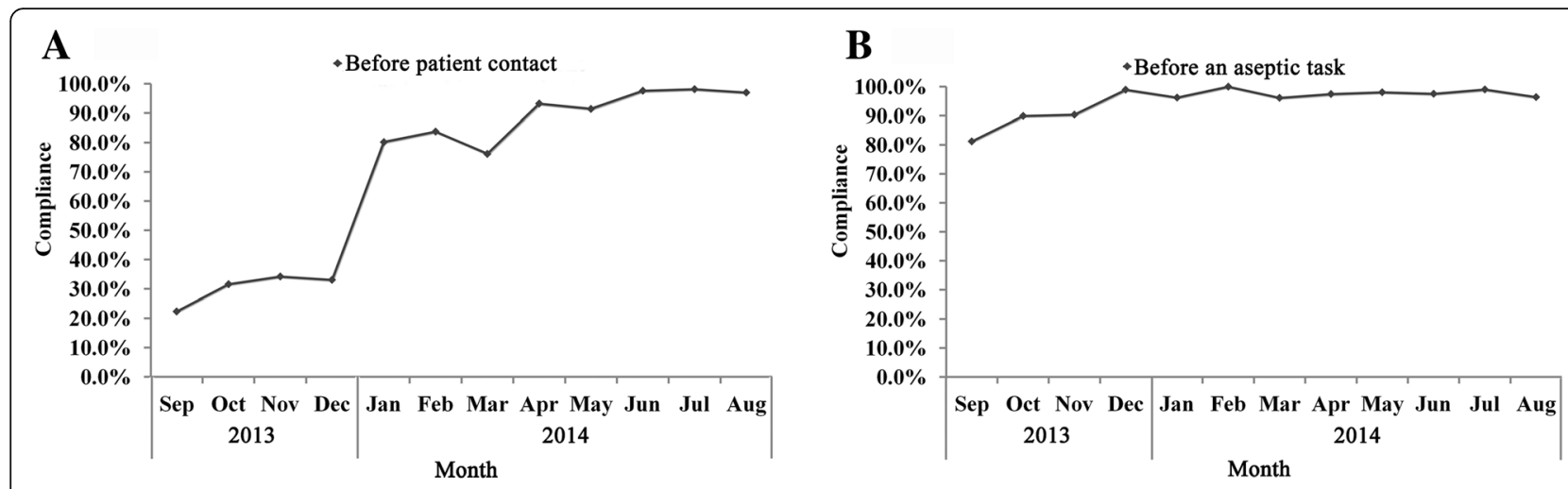

C
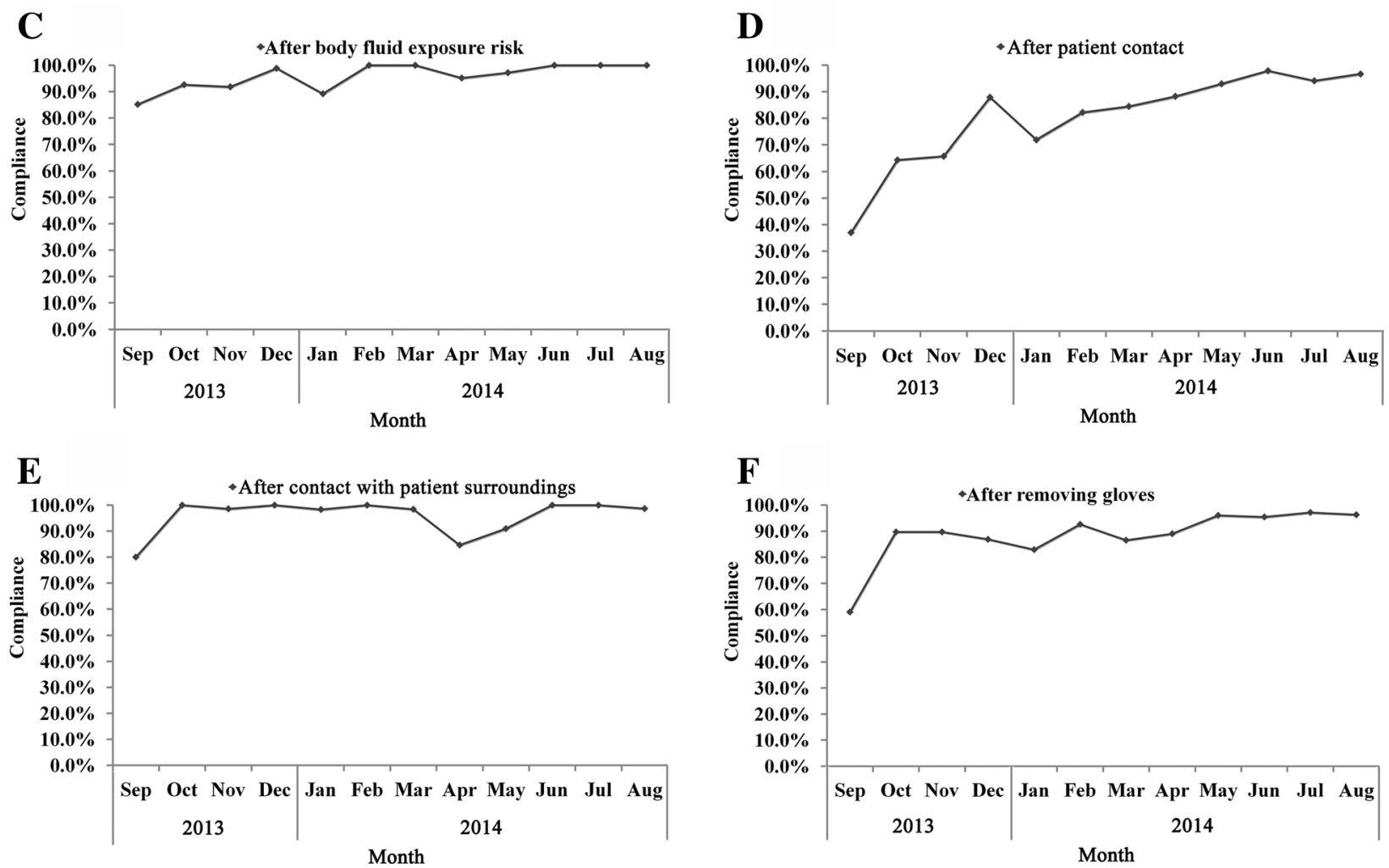

Fig. 3 Monthly hand hygiene compliance of before patient contact (a), before an aseptic task (b), after body fluid exposure risk (c), after patient contact (d), after contact with patient surroundings (e) and after removing gloves ( $\mathbf{f}$ )

compliance. Though training is the most basic intervention element, surveillance, evaluation and feedback should be explored as additional interventions to ensure that hand hygiene compliance is achieved and sustained at high levels.

\section{Additional files}

Additional file 1: The basic steps and approximate schedule of quality control circle (QCC) program. (DOC $32 \mathrm{~kb}$ )

Additional file 2: The major agenda and interventions proposed in circle meetings during the period of quality control circle (QCC) program. (DOCX $15 \mathrm{~kb}$ )
Additional file 3: The process and basic data of Cochran Armitage trend test applied for the linear trend of hand hygiene compliance. (DOCX $29 \mathrm{~kb})$

Abbreviations

HAI: Healthcare-associated infection; HCWs: Healthcare workers; PDCA: Plan-do-check-act; QCC: Quality control circle; WHO: World Health Organization

\section{Acknowledgments}

We would like to thank the staff in department of implant dentistry in Stomatology Hospital of Shandong University for their participation in this QCC program. 


\section{Funding}

This study was supported by a grant from Taishan Scholar Program of Shandong Province (ts.201511105)

\section{Availability of data and materials}

The datasets supporting the results of this article are included within the article and its Additional files 1, 2 and 3.

\section{Authors' contributions}

PC, TY and AX conceived the study and drafted the paper. QS, LJ and ZZ coordinated and performed the data collection. HJ contributed to the conception and design of the study. ZT and HW provided critical revision of the manuscript. All authors read and approved the final manuscript.

\section{Competing interests}

The authors declare that they have no competing interests.

\section{Consent for publication}

Not applicable.

\section{Ethics approval and consent to participate}

Our study was approved by the Research Ethic Committee of Shandong University Dental School (No. 201309). All the QCC members were informed and voluntarily willing to participate in the hand hygiene QCC program.

\section{Author details}

'Department of Epidemiology, School of Public Health, Shandong University, Jinan, Shandong, People's Republic of China. ${ }^{2}$ Shandong Provincial Key Laboratory of Oral Tissue Regeneration, Shandong University, Jinan, Shandong, People's Republic of China. ${ }^{3}$ School of Stomatology, Shandong University, Jinan, Shandong, People's Republic of China. ${ }^{4}$ Department of General Medicine, Shandong Provincial Hospital Affiliated to Shandong University, Jinan, Shandong, People's Republic of China. ${ }^{5}$ Shandong Provincial Key Laboratory of Infectious Disease Control and Prevention, Jinan, Shandong, People's Republic of China. 'Shandong Center for Disease Control and Prevention, No. 16992 Jingshi Road, Jinan 250014, Shandong, People's Republic of China.

Received: 14 October 2016 Accepted: 1 December 2016

Published online: 08 December 2016

\section{References}

1. Wensing M, Broge B, Riens B, Kaufmann-kolle P, Akkermans R, Grol R, et al, Quality circles to improve prescribing of primary care physicians. Three comparative studies. Pharmacoepidemiol Drug Saf. 2009;18:763-9.

2. Pethyoung $W$, Picheansathian $W$, Boonchuang $P$, Apisarnthanarak $A$ Danchaivijitr S. Effectiveness of education and quality control work group focusing on nursing practices for prevention of ventilator-associated pneumonia. J Med Assoc Thai. 2005;Suppl 10:S110-4.

3. Spiegel W, Mlczoch-Czerny MT, Jens R, Dowrick C. Quality circles for pharmacotherapy to modify general practitioners' prescribing behaviour for generic drugs. J Eval Clin Pract. 2012;18:828-34.

4. Chen W, Deng H, Shen L, Qin M, He L. A comprehensive intervention program on the long-term placement of peripherally inserted central venous catheters. J Cancer Res Ther. 2014;10:359-62.

5. Chang YN, Lin LH, Chen WH, Liao HY, Hu PH, Chen SF, et al. Quality control work group focusing on practical guidelines for improving safety of critically ill patient transportation in the emergency department. J Emerg Nurs. 2010;36:140-5.

6. Pan SC, Tien KL, Hung IC, Lin YJ, Sheng WH, Wang MJ, et al. Compliance of health care workers with hand hygiene practices: independent advantages of overt and covert observers. PLOS ONE. 2013;8:e53746.

7. Musuuza JS, Barker A, Ngam C, Vellardita L, Safdar N. Assessment of fidelity in interventions to improve hand hygiene of healthcare workers: a systematic review. Infect Control Hosp Epidemiol. 2016;10:1-9.

8. World Health Organization. WHO Guidelines on Hand Hygiene in Health Care: First Global Patient Safety Challenge, Clean Care is Safer Care. Geneva: WHO; 2009.

9. Pittet D, Simon A, Hugonnet S, Pessoa-Silva CL, Sauvan V, Perneger TV. Hand hygiene among physicians: performance, beliefs, and perceptions. Ann Intern Med. 2004;141:1-8.
10. Erasmus V, Daha TJ, Brug H, Richardus JH, Behrendt MD, Vos MC, et al. Systematic review of studies on compliance with hand hygiene guidelines in hospital care. Infect Control Hosp Epidemiol. 2010;31:283-94.

11. Scheithauer $S$, Rosarius A, Rex S, Post P, Heisel H, Krizanovic V, et al. Improving hand hygiene compliance in the anesthesia working room work area: More than just more hand rubs. Am J Infect Control. 2013:41:1001-6.

12. Wang LR, Wang Y, Lou Y, Li Y, Zhang XG. The role of quality control circles in sustained improvement of medical quality. SpringerPlus. 2013;2:141.

13. Loveday HP, Lynam S, Singleton J, Wilson J. Clinical glove use: healthcare workers' actions and perceptions. J Hosp Infect. 2014;86:110-6.

14. Han K, Dou FM, Zhang LJ, Zhu BP. Compliance on hand-hygiene among healthcare providers working at secondary and tertiary general hospitals in Chengdu. Zhonghua Liu Xing Bing Xue Za Zhi. 2011;32:1139-42.

15. Liu WI, Liang SY, Wu SF, Chuang YH. Hand hygiene compliance among the nursing staff in freestanding nursing homes in Taiwan: a preliminary study. Int J Nurs Pract. 2014;20:46-52.

16. Lau T, Tang G, Mark KL, Leung G. Moment-specific compliance with hand hygiene. Clin Teach. 2014;11:159-64.

17. Eckmanns T, Bessert J, Behnke M, Gastmeier P, Ruden H. Compliance with antiseptic hand rub use in intensive care units: the Hawthorne effect. Infect Control Hosp Epidemiol. 2006;27:931-4.

18. Hagel S, Reischke J, Kesselmeier M, Winning J, Gastmeier P, Brunkhorst FM, et al. Quantifying the Hawthorne Effect in hand hygiene compliance through comparing direct observation with automated hand hygiene monitoring. Infect Control Hosp Epidemiol. 2015;36:957-62.

19. Dhar S, Tansek R, Toftey EA, Dziekan BA, Chevalier TC, Bohlinger CG, et al. Observer bias in hand hygiene compliance reporting. Infect Control Hosp Epidemiol. 2010;31:869-70.

20. Maury E, Moussa N, Lakermi C, Barbut F, Offenstadt G. Compliance of health care workers to hand hygiene: awareness of being observed is important. Intensive Care Med. 2006:32:2088-9.

\section{Submit your next manuscript to BioMed Central and we will help you at every step:}

- We accept pre-submission inquiries

- Our selector tool helps you to find the most relevant journal

- We provide round the clock customer support

- Convenient online submission

- Thorough peer review

- Inclusion in PubMed and all major indexing services

- Maximum visibility for your research

Submit your manuscript at www.biomedcentral.com/submit
Biomed Central 